\title{
SBOLme: a repository of SBOL parts for metabolic engineering
}

\author{
Hiroyuki Kuwahara, ${ }^{\dagger}, \|$ Xuefeng Cui, ${ }^{\dagger},+\|$ Ramzan Umarov, ${ }^{\dagger}$ Raik Grünberg,, \\ Chris J. Myers, ${ }^{\S}$ and Xin Gao, ${ }^{*}$ \\ $\dagger$ †ing Abdullah University of Science and Technology (KAUST), Computational Bioscience \\ Research Center (CBRC), Thuwal, 23955, Saudi Arabia \\ $\ddagger$ Current Address: Institute for Interdisciplinary Information Sciences (IIIS), Tsinghua \\ University, Beijing, 100084, China \\ \King Abdullah University of Science and Technology (KAUST), Biological and \\ Environmental Science and Engineering Division, Thuwal, 23955, Saudi Arabia \\ $\S$ Department of Electrical and Computer Engineering, University of Utah, Salt Lake City, \\ Utah, 84112, USA \\ \|Equal contributions \\ E-mail: xin.gao@kaust.edu.sa \\ Phone: +966 12808 0323. Fax: +966 128021241
}

\begin{abstract}
The Synthetic Biology Open Language (SBOL) is a community-driven open language to promote standardization in synthetic biology. To support the use of SBOL in metabolic engineering, we developed SBOLme, the first open-access repository of SBOL 2.0-compliant biochemical parts for a wide range of metabolic engineering applications. The URL of our repository is http://www.cbrc.kaust.edu.sa/sbolme.
\end{abstract}




\section{Keywords}

metabolic engineering, synthetic biology, standardization, parts repository

\section{Introduction}

Standardization of biological parts is a cornerstone in synthetic biology as it allows for effective communication of biological designs, which in turn makes the engineering of complex biological systems more efficient. The Synthetic Biology Open Language (SBOL) is a community-driven standard to specify the design of synthetic biological systems (1). As the SBOL standard promotes an efficient means to exchange and re-use designs (2), it was adopted by many experimental and computational synthetic biologists early on. In line with this development, ACS Synthetic Biology recently updated its guidance to officially adopt the SBOL standard and recommend the use of SBOL $2.0(3,4)$ to its authors $(5)$. Despite these community efforts to advocate the SBOL standard in synthetic biology, strong support for its use has been, so far, lacking for applications focusing on metabolic engineering. To promote the use of standards in metabolic engineering, we developed SBOLme, an openaccess repository of SBOL 2.0-compliant (3) metabolic parts. To our knowledge, SBOLme is the first repository to cover a wide range of SBOL 2.0-compliant metabolic parts.

\section{SBOLme}

\subsection{Types of metabolic parts}

SBOLme contains four different types of metabolic parts: chemical compounds; enzyme classes in the Enzyme Commission (EC) number; metabolic proteins; and metabolic reactions (Figure 1). In the initial release, the SBOLme repository has metabolic SBOL parts from 3,908 organisms and includes 28,437 chemical compounds, 6,883 EC numbers, 3,173,238 metabolic proteins, and 9,909 metabolic reactions (Figure 1). We constructed 
these metabolic parts and validated their SBOL 2.0 compliance using libSBOLj $(6)$.

\subsection{Chemical compounds}

SBOLme contains chemical compound parts that are from the KEGG COMPOUND and GLYCAN databases. Chemical compounds are represented by the ComponentDefinition class. They have references to compound databases such as PubChem (7), ChEBI (8), and KEGG (9). Among the compound parts in SBOLme, 1,386 are drug compounds, which are annotated with information about interacting proteins given in DrugBank (10). To enable thermodynamics-based analysis, the chemical compound type includes information about the standard Gibbs free energy of formation from eQuilibrator (11). To include SMILES sequences information in the chemical compound parts, we made use of the PubChem and ChEBI databases by applying the mapping from the KEGG COMPOUND ID to the PubChem and ChEBI IDs. With this approach, we were able to provide SMILES sequences for 16,129 compounds.

\section{$2.3 \quad$ EC numbers}

To describe a metabolic design without specifying actual enzymes in enzymatic reactions, we constructed metabolic parts that specify abstract classes of enzymes based on their EC numbers. The type of these parts are based on EC number which is a hierarchical classification scheme which categorizes enzymes based on chemical transformations that they catalyze. The EC number parts are represented by ComponentDefinitions, and they are annotated with their chemical transformations using ExPASy ENZYME (12).

\subsection{Metabolic proteins}

Metabolic proteins are also represented by ComponentDefinitions. Most of these proteins $(3,172,324$ out of $3,173,238$ proteins) are enzymes that catalyze chemical transformations. 
Out of these enzymes, 2,939,191 are annotated with complete EC numbers and can be connected to the corresponding EC number parts. Some of these parts (1,000 proteins) are also annotated with their catalytic site information using Catalytic Site Atlas (13), which may facilitate protein design. These catalytic site links were established through the mapping from UniProt IDs (14) in Catalytic Site Atlas to the corresponding KEGG GENE IDs. The other protein parts included in SBOLme are those involved in interactions with drug compounds such as carriers and transporters. These drug-interaction-related proteins (2,380 proteins) are referenced by at least one drug compound in the repository. These links

were created by mapping UniProt IDs in DrugBank entries to the corresponding KEGG IDs. Protein parts have references to protein databases such as UniProt (14), PDB (15), NCBI (16) and KEGG (9).

\subsection{Metabolic reactions}

Metabolic reactions describe biochemical transformations of compounds, and they are represented by the ModuleDefinition class. Reaction parts act as glue to connect chemical compounds and to form chemical transformation pathways. In this release, 8,006 out of the 28,437 chemical compound parts are connected to reaction parts. Most of the reaction parts $(8,637$ out of 9,909$)$ represent enzymatic reactions. Out of these enzymatic reaction parts, 7,564 are annotated with complete EC numbers. In total, these enzymatic reaction parts are connected to 4,798 different EC number parts. Reaction parts contain annotations of their chemical transformations such as reaction stoichiometry. They are also annotated with the information about the standard Gibbs free energy of reaction from eQuilibrator (11). This link was established using the KEGG REACTION IDs that are present in the eQuilibrator data set. With the MapsTo property in the Module class, the user can specify an enzymatic reaction with different levels of abstraction; a detailed design of a metabolic reaction can be described by linking a metabolic reaction to a specific enzyme, while a more abstracted design can be communicated by linking a metabolic reaction to a specific EC number. 


\subsection{Metabolic parts exploration}

With the SBOLme web server, the user can explore the aforementioned metabolic parts through a catalog for each type. The user can also query these parts based on various criteria. This includes parts query based on their names and IDs as well as their functional and structural/sequence features. For example, by specifying a metabolic reaction, the user can search for chemical compounds participating in this reaction, while, by specifying a compound substructure in SMILES, the user can search for compounds containing this substructure. In the case of a search for metabolic enzymes, the user can specify query criteria based on a combination of an EC number, an amino acid sequence, and an organism. These query features allow the user to more conveniently look for appropriate SBOL-compliant metabolic parts and efficiently construct designs for biosynthesis systems.

\subsection{Pathway design using SBOLme parts}

To illustrate how SBOLme parts can be used in the design of metabolic pathways, we present here a simple biosynthesis design example which produces naringenin from p-coumaroyl-CoA, as shown in Figure 2. To search for this naringenin pathway, we applied MRE (17) with the host organism 'Escherichia coli K-12 MG1655,' the starting material 'p-coumaroyl-CoA,' and the desired product 'naringenin.' With the default setting, we obtained a pathway based on two enzymatic reactions: naringenin-chalcone synthase (EC:2.3.1.74) and chalcone isomerase (EC:5.5.1.6). Since these two classes of enzymes are not available in E. coli, MRE suggested foreign enzymes: AT5G13930 for EC:2.3.1.74 and AT3G55120 for EC:5.5.1.6 from Arabidopsis thaliana. Based on these, we obtained metabolic parts from the SBOLme website necessary to make two pathway designs at different levels of details: an abstracted design with only enzyme classes specified for the reactions and a detailed design with actual enzymes specified as the catalysts of those reactions (Figure 2). These pathways along with the metabolic parts used in them are available for download from Supporting Information. 


\section{Discussions}

Here, we have introduced SBOLme, the first open-access repository of SBOL 2.0-compliant metabolic parts. Since many applications of synthetic biology focus on biosynthesis of highvalue natural products (e.g., the production of biofuels, cosmetics, perfumes, and drugs) (18), synthetic biologists would benefit greatly with a strong support for the use of the community-driven standard for such applications. Thus, the new repository is expected to facilitate the development of an infrastructure in which synthetic biologists can more effectively conceptualize and communicate their designs for biosynthesis systems.

Our future work includes further validation of our integrated data, the development of client data-access APIs such as those based on the SBOL stack (19), and the integration of SBOLme with biosynthesis pathway design tools such as MRE (17). SBOLme can be accessed at http://www.cbrc.kaust.edu.sa/sbolme. Data accessed through the SBOLme web server are provided under the Creative Commons 4.0 license.

\section{Acknowledgement}

This study is based upon work supported by the King Abdullah University of Science and Technology (KAUST) Office of Sponsored Research (OSR) under Award No URF/1/1976-04 and by the National Science Foundation under Grants CCF-1218095 and DBI-135604. Any opinions, findings, and conclusions or recommendations expressed in this material are those of the author(s) and do not necessarily reflect the views of the National Science Foundation.

\section{Supporting Information Available}

The following files are available free of charge.

- supp1.zip: a zip file containing SBOL files for the metabolic parts and pathways used in Figure 2. 


\section{References}

1. Galdzicki, M., Clancy, K. P., Oberortner, E., Pocock, M., Quinn, J. Y., Rodriguez, C. A., Roehner, N., Wilson, M. L., Adam, L., and Anderson, J. C. et al. (2014) The Synthetic Biology Open Language (SBOL) provides a community standard for communicating designs in synthetic biology. Nat Biotechnol 32, 545-550.

2. Roehner, N., Beal, J., Clancy, K., Bartley, B., Misirli, G., Grünberg, R., Oberortner, E., Pocock, M., Bissell, M., and Madsen, C. et al. (2016) Sharing Structure and Function in Biological Design with SBOL 2.0. ACS Synth Biol 5, 498-506.

3. Bartley, B., Beal, J., Clancy, K., Misirli, G., Roehner, N., Oberortner, E., Pocock, M. R., Bissell, M., Madsen, C., and Nguyen, T. et al. (2015) Synthetic Biology Open Language (SBOL) Version 2.0.0. J Integr Bioinform 12.

4. Beal, J., Cox, R., Grünberg, R., McLaughlin, J., Nguyen, T., Bartley, B., Bissell, M., Choi, K., Clancy, K., and Macklin, C. et al. (2016) Synthetic Biology Open Language (SBOL) Version 2.1.0. J Integr Bioinform 13.

5. Hillson, N. J., Plahar, H. A., Beal, J., and Prithviraj, R. (2016) Improving Synthetic Biology Communication: Recommended Practices for Visual Depiction and Digital Submission of Genetic Designs. ACS Synth Biol 5, 449-451.

6. Zhang, Z., Nguyen, T., Roehner, N., Misirli, G., Pocock, M., Oberortner, E., Samineni, M., Zundel, Z., Beal, J., and Clancy, K. et al. (2015) libSBOLj 2.0: A Java Library to Support SBOL 2.0. IEEE Life Sci. Lett. 1, 34-37.

7. Kim, S., Thiessen, P. A., Bolton, E. E., Chen, J., Fu, G., Gindulyte, A., Han, L., He, J., He, S., and Shoemaker, B. A. et al. (2016) PubChem Substance and Compound databases. Nucleic Acids Res 44, D1202-D1213. 
8. Degtyarenko, K., De Matos, P., Ennis, M., Hastings, J., Zbinden, M., McNaught, A., Alcántara, R., Darsow, M., Guedj, M., and Ashburner, M. (2008) ChEBI: a database and ontology for chemical entities of biological interest. Nucleic Acids Res 36, D344-D350.

9. Kanehisa, M., Goto, S., Sato, Y., Kawashima, M., Furumichi, M., and Tanabe, M. (2014) Data, information, knowledge and principle: back to metabolism in KEGG. Nucleic Acids Res 42, D199-D205.

10. Wishart, D. S., Knox, C., Guo, A. C., Shrivastava, S., Hassanali, M., Stothard, P., Chang, Z., and Woolsey, J. (2006) DrugBank: a comprehensive resource for in silico drug discovery and exploration. Nucleic Acids Res 34, D668-D672.

11. Flamholz, A., Noor, E., Bar-Even, A., and Milo, R. (2012) eQuilibrator-the biochemical thermodynamics calculator. Nucleic Acids Res 40, D770-D775.

12. Bairoch, A. (2000) The ENZYME database in 2000. Nucleic Acids Res 28, 304-305.

13. Furnham, N., Holliday, G. L., de Beer, T. A. P., Jacobsen, J. O. B., Pearson, W. R., and Thornton, J. M. (2014) The Catalytic Site Atlas 2.0: cataloging catalytic sites and residues identified in enzymes. Nucleic Acids Res 42, D485-D489.

14. Consortium, U. (2015) UniProt: a hub for protein information. Nucleic Acids Res 43, D204-D212.

15. Berman, H. M., Westbrook, J., Feng, Z., Gilliland, G., Bhat, T. N., Weissig, H., Shindyalov, I. N., and Bourne, P. E. (2000) The Protein Data Bank. Nucleic Acids Res. 28, 235-242.

16. Geer, L. Y., Marchler-Bauer, A., Geer, R. C., Han, L., He, J., He, S., Liu, C., Shi, W., and Bryant, S. H. (2010) The NCBI BioSystems database. Nucleic Acids Res 38, D492D496. 
17. Kuwahara, H., Alazmi, M., Cui, X., and Gao, X. (2016) MRE: a web tool to suggest foreign enzymes for the biosynthesis pathway design with competing endogenous reactions in mind. Nucleic Acids Res 44, W217-W225.

18. Smanski, M. J., Zhou, H., Claesen, J., Shen, B., Fischbach, M. A., and Voigt, C. A. (2016) Synthetic biology to access and expand nature's chemical diversity. Nat Rev Microbiol $14,135-149$.

19. Madsen, C., McLaughlin, J. A., Misırlı, G., Pocock, M., Flanagan, K., Hallinan, J., and Wipat, A. (2016) The SBOL Stack: A Platform for Storing, Publishing, and Sharing Synthetic Biology Designs. ACS Synth Biol 5, 487-497. 


\section{Figures}

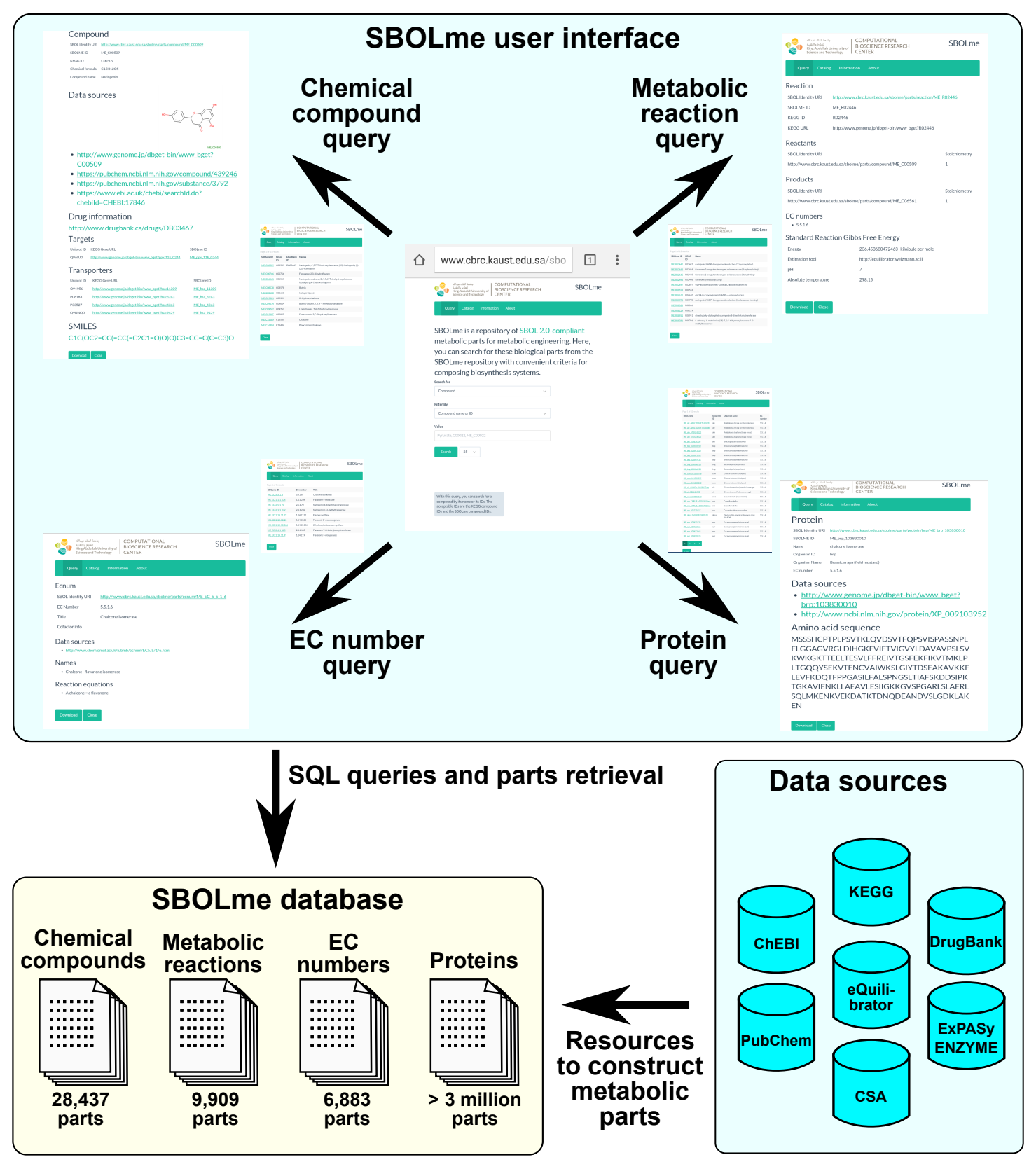

Figure 1: SBOLme user interface and parts repository. The user can access to SBOLme at http://www.cbrc.kaust.edu.sa/sbolme. The web site has a user interface which allows the user to search for chemical compound, metabolic reaction, EC number, and protein parts. These four different types of metabolic parts all are stored in the backend database. Each of the parts was constructed by compiling data from various sources as shown here. 
SBOLme webserver

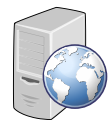

Metabolic parts repository

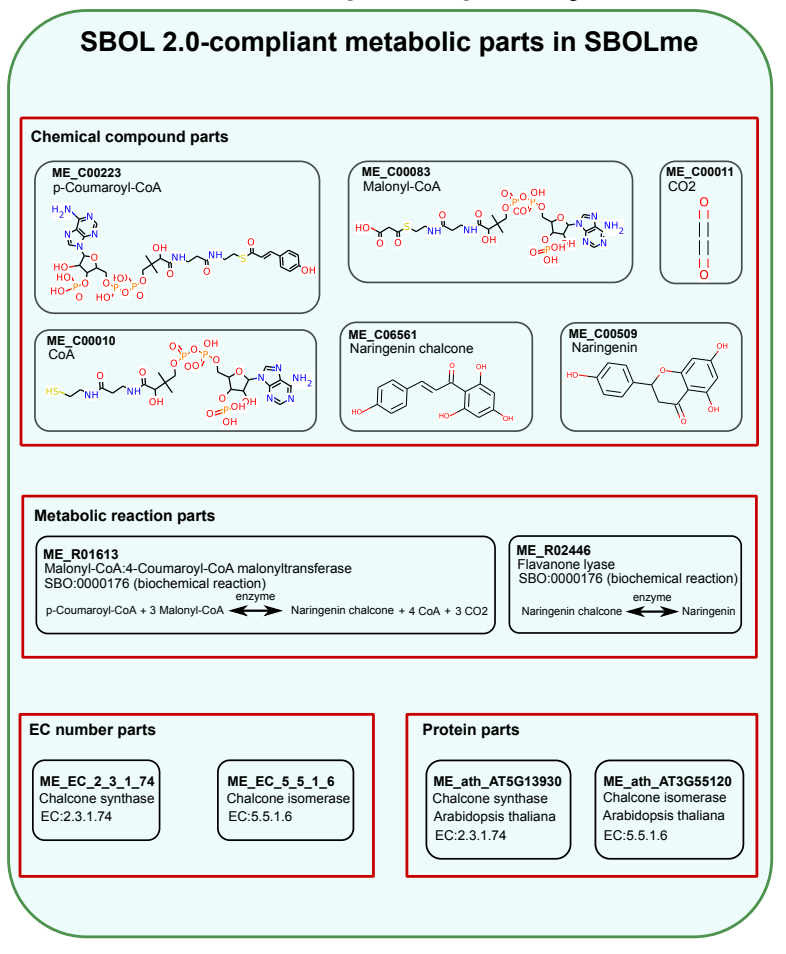

SBOL-compliant design tool
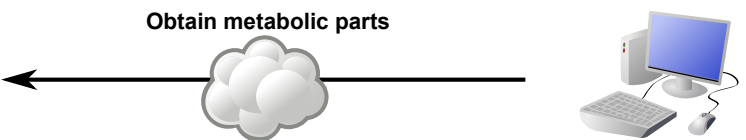

Biosynthesis system design

Biosynthesis of naringenin from p-coumaroyl-CoA

Abstracted design with only enzyme classes specified

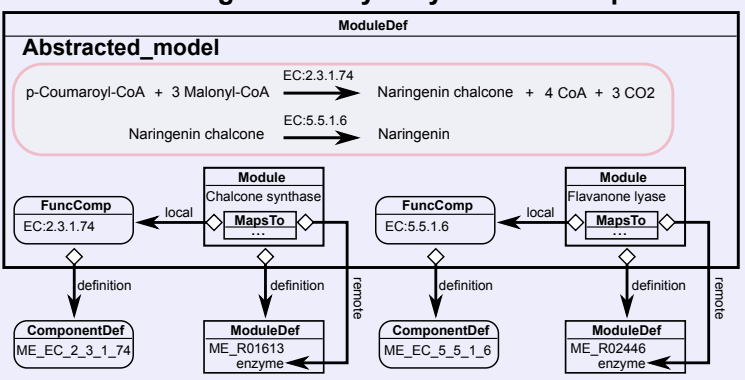

Detailed design with actual enzymes specified

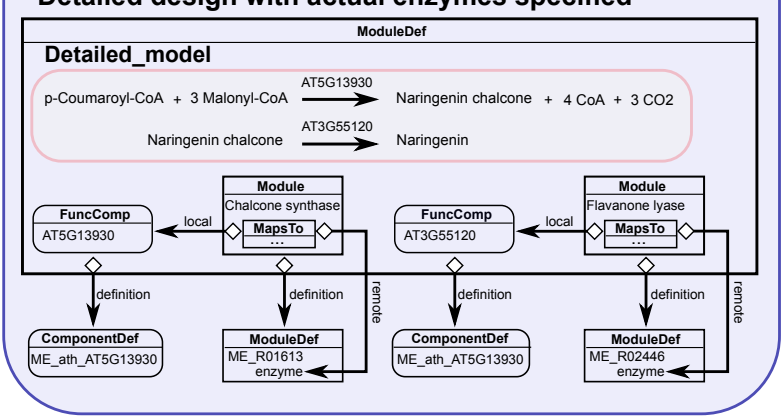

Figure 2: An illustration of the use of SBOLme metabolic parts for the design of biosynthesis systems by using the synthesis of naringenin from p-coumaroyl-CoA as an example. Here, the left pane shows metabolic parts that can be used to construct a naringenin-biosynthesis pathway, while the right pane shows two different levels of designs of the biosynthesis pathway using SBOL. The first design shows an indeterminate design with only enzyme classes specified for the metabolic reactions. The second one shows a more complete design with actual enzymes specified for the metabolic reactions. In this figure, ModuleDef, ComponentDef, and FuncComp are ModuleDefinition, ComponentDefinition, and FunctionalComponent, respectively. SBOL files for the parts and pathways in this example are included in supplementary materials. 